\title{
Is Less More: Does Leukopenia Predict Remission in Patients with Inflammatory Bowel Disease Receiving Thiopurine Treatment?
}

\author{
Javier P. Gisbert
}

Published online: 10 December 2014

(C) Springer Science+Business Media New York 2014

The thiopurine drugs azathioprine (AZA) and mercaptopurine (MP) effectively maintain remission in patients with inflammatory bowel disease (IBD) [1]. Dosing of AZA and MP is generally weight based, with the intention of achieving the highest therapeutic efficacy and, at the same time, reducing the incidence of adverse effects. Several strategies have been proposed to optimally dose thiopurines with the intention, on the one hand, to identify patients at risk of adverse effects (mainly myelotoxicity) and, on the other hand, to detect patients with subtherapeutic doses and inadequate immunosuppression [2]. These strategies include monitoring changes in the red cell mean corpuscular volume, induction of leukopenia, quantification of 6-thioguanine nucleotides (6-TGN), and the monitoring of thiopurine methyltransferase (TPMT) activity.

Mild or chronic leukopenia, generally defined as a white cell count between 3,000 and $4,000 / \mu \mathrm{L}$, is the commonest hematologic effect of thiopurines, appearing in 5-25\% of the patients receiving these drugs [3]. The incidence rate of myelotoxicity in IBD patients receiving AZA/MP has been calculated, in a recent systematic review, to be approximately $3 \% /$ patient-year of treatment [3]. Although leukopenia is a well-recognized effect of AZA/MP treatment, its association with therapeutic efficacy has yet to be determined, although the induction of leukopenia is probably closely related to the therapeutic mechanisms of thiopurines.

\section{J. P. Gisbert $(\bowtie)$}

Gastroenterology Unit, Instituto de Investigación Sanitaria Princesa and Centro de Investigación Biomédica en Red de Enfermedades Hepáticas y Digestivas (CIBEREHD), Hospital Universitario de La Princesa, Playa de Mojácar 29, Urb. Bonanza, 28669 Boadilla del Monte, Madrid, Spain e-mail: javier.p.gisbert@gmail.com
In this context, the aim of the study by Park et al. [4] published in this issue of Digestive Diseases and Sciences was to evaluate the correlation between thiopurine-induced leukopenia and clinical outcomes in Korean IBD patients who achieved clinical remission with thiopurines. The authors included 196 IBD patients [45 with ulcerative colitis (UC), 68 with Crohn's disease (CD), and 83 with intestinal Behçet's disease] who were treated with AZA/ MP and had achieved remission. Then, the authors retrospectively analyzed the lowest white blood cell (WBC) count during AZA/MP treatment, duration of remission, and the occurrence of relapse and compared the clinical evolution between leukopenic (WBC count $<4,000 / \mu \mathrm{L}$ ) and non-leukopenic patients. The authors reported that the cumulative relapse-free survival rate was higher in the leukopenic group. Thus, relapse occurred in $31 \%$ of leukopenic patients and in $54 \%$ of non-leukopenic patients $(P=0.001)$. Using multivariate analysis, the presence of leukopenia was inversely associated with relapse. Therefore, the authors concluded that leukopenia occurring during thiopurine maintenance therapy is associated with prolonged remission in patients with IBD, including those with Behçet's disease.

Although undoubtedly interesting and clinically relevant, this study has several limitations that should be taken into account:

1. The first and obvious shortcoming is the retrospective design of the study.

2. Korean patients seem to become leukopenic at a higher rate than Western patients. Compared with previous reports based on Western populations, a higher percentage of leukopenia (61\%) was reported in this study. Although the reason for these regional differences is not clear, it may be due to different 
TPMT genetic polymorphisms between Asian and Western populations. These differences in any case call for caution before extrapolating the results of this study to Western populations.

3. As an inclusion criterion, it was required that the duration of thiopurine therapy was $\geq 1$ month. Since a longer time period (up to 4 months) may be needed before the full therapeutic benefit of thiopurines can be realized, it is possible that some patients could have been classified as non-responders while they, in fact, may have been late thiopurine responders.

4. The authors only enrolled patients who had achieved clinical remission after thiopurine therapy. This study evaluates the relationship between thiopurine-induced leukopenia and clinical outcomes in patients who have already achieved clinical remission with thiopurines, thus biasing the results to thiopurine responders. The study does not estimate the probability of achieving remission in previously untreated patients, which include thiopurine nonresponders.

5. The study also excluded patients who were lost to follow-up and patients with poor drug compliance. These exclusion criteria should be considered before extrapolating the results of this study to clinical practice, where compliance with treatment (and specifically with thiopurines) may be a concern.

6. The threshold for defining leukopenia was stated at $4,000 \mathrm{WBC} / \mu \mathrm{L}$, which is probably too high. Although the definition of drug-induced leukopenia varies markedly among published studies, the cutoff point for the number of leukocytes was generally set at $3,000-4,000 / \mu \mathrm{L}$. Using the definition used by Park et al. (WBC $<4,000 \mathrm{WBC} / \mu \mathrm{L}$ ), more than half $(61 \%)$ of patients included in the study eventually became leukopenic during thiopurine therapy and had to take a lower than recommended dose. Approximately $50 \%$ of them had $3,000-4,000 \mathrm{WBC} / \mu \mathrm{L}$, while the other $50 \%$ had $2,000-3,000 \mathrm{WBC} / \mu \mathrm{L}$. Finally, it may perhaps be more important to consider the percentage decrease of WBC after prescribing thiopurine drugs rather than the absolute number of leukocytes.

7. Immunosuppression by thiopurines is theoretically mediated through lymphocytes. Nonetheless, as the authors state, neutrophils are preferentially suppressed when leukopenia occurs, suggesting that the anti-inflammatory effects of thiopurines are mediated through neutrophils as well [5], which agrees well with the observation that clinical relapse of IBD is characterized by increased neutrophil migration into the intestine. Rather, the propensity to infection in neutropenic patients is related to the absolute neutrophil count and the duration of neutropenia [3]; thus, in patients with a neutrophil count $>1,500 / \mu \mathrm{L}$, no significant risk of infection exists [3]. Therefore, neutropenia, rather than leukopenia, may be a more important hematologic value to measure.

8. The authors state that if leukopenia occurred, the dose of AZA/MP was reduced or the therapy was withdrawn. Nevertheless, data regarding the way thiopurines were restarted were not provided. In the Discussion, the authors concluded that thiopurineinduced mild leukopenia $(3,000 \leq \mathrm{WBC}<4,000$ / $\mu \mathrm{L})$ is a safe value at which to maintain remission, but the authors stated in the Methods section that if leukopenia occurred, the dose of AZA/6-MP was reduced or the therapy was withdrawn until the WBC count recovered to $>4,000 / \mu \mathrm{L}$.

9. Higher doses of AZA (2.5 mg/kg) are more effective than lower doses $(1.0$ or $2.0 \mathrm{mg} / \mathrm{kg}$ ) for preventing IBD relapse [1]. In the present study, patients were initially administered AZA/MP at $1.0 \mathrm{mg} / \mathrm{kg}$ daily. If that was well tolerated without adverse effects, the dose was gradually increased every 2-4 weeks until patients reached a dose of $2.0-2.5 \mathrm{mg} / \mathrm{kg}$. However, the median AZA/MP maintenance dose was only $1.6 \mathrm{mg} / \mathrm{kg}$ (although the corresponding doses for AZA and MP were not provided separately). These low thiopurine doses may, at least partially, explain the high incidence of relapses reported in the study (31\% of leukopenic patients and in $54 \%$ of nonleukopenic patients), which contrast with the lower relapse figures reported in the literature [1].

10. Leukopenia has been reported to be more common in patients receiving thiopurines and 5-ASA compared with those receiving thiopurines alone [6]. The high incidence of leukopenia in the study by Park et al. might have resulted from the high percentage (98\%) of concurrent 5-aminosalicyclic acid (5-ASA) use during thiopurine therapy. This very high percentage of 5-ASA prescription is surprising, as more than half of IBD patients included in the study had CD, a disease in which 5-ASA treatment is controversial at best [7].

11. Low TPMT activity and high 6-TGN concentrations have been linked to therapeutic success and myelosuppression in IBD patients treated with thiopurines $[3,8]$. Yet, none of these metabolites or activities was measured in the study. Nevertheless, the efficacy of the strategy of determining TPMT activity or genotype and/or 6-TGN concentrations in all patients prior to initiating treatment with thiopurine drugs has not yet been definitively confirmed.

12. An additional limitation of the study by Park et al. is that the definition of remission and relapse was 
based solely on clinical symptoms, while it is widely accepted that between clinical manifestations and endoscopic findings correlate poorly in IBD patients. That is why, at present, endoscopic remission is increasingly considered as the "gold standard" for defining remission in patients with IBD.

Finally, although it is true that another report has suggested that thiopurine-induced leukopenia is associated with lower relapse rates and prolonged remission in IBD patients [9], more recent studies indicate that thiopurineinduced leukopenia does not predict the relapse rate of IBD [10-12].

In conclusion, as the authors recognize, correlation between thiopurine-induced leukopenia and clinical outcomes of IBD still remains uncertain. Therefore, further studies-ideally prospective randomized controlled trials-should be conducted to confirm whether leukopenia (or neutropenia) occurring during thiopurine maintenance therapy is associated with prolonged remission in patients with IBD and whether this strategy may be useful in clinical practice.

\section{References}

1. Prefontaine E, Sutherland LR, Macdonald JK, Cepoiu M. Azathioprine or 6-mercaptopurine for maintenance of remission in Crohn's disease. Cochrane Database Syst Rev. 2009;1:CD000067.

2. Gisbert JP, Gomollon F, Mate J, Pajares JM. Individualized therapy with azathioprine or 6-mercaptopurine by monitoring thiopurine methyl-transferase (TPMT) activity. Rev Clin Esp. 2002;202:555-562.

3. Gisbert JP, Gomollon F. Thiopurine-induced myelotoxicity in patients with inflammatory bowel disease: a review. Am J Gastroenterol. 2008;103:1783-1800.

4. Park MS, Kim DH, Kim DH, et al. Leukopenia predicts remission in patients with inflammatory bowel disease and Behcet's disease on thiopurine maintenance. Dig Dis Sci. (Epub ahead of print). doi:10.1007/s10620-014-3355-4.

5. Korelitz BI, Zlatanic J, Smith MJ, Lipe RJ, Baiocco PJ, Gleim GW. Significance of WBC differential when leukopenia is induced by 6-MP for IBD. Gastroenterology. 1997;113: 1810-1811.

6. Lowry PW, Franklin CL, Weaver AL, et al. Leucopenia resulting from a drug interaction between azathioprine or 6-mercaptopurine and mesalamine, sulphasalazine, or balsalazide. Gut. 2001;49:656-664.

7. Gisbert JP, Chaparro M, Gomollon F. Common misconceptions about 5-aminosalicylates and thiopurines in inflammatory bowel disease. World J Gastroenterol. 2011;17:3467-3478.

8. Gisbert JP, Gomollon F, Cara C, et al. Thiopurine methyltransferase activity in Spain: a study of 14,545 patients. Dig Dis Sci. 2007;52:1262-1269.

9. Colonna T, Korelitz BI. The role of leukopenia in the 6-mercaptopurine-induced remission of refractory Crohn's disease. Am J Gastroenterol. 1994;89:362-366.

10. Campbell S, Ghosh S. Is neutropenia required for effective maintenance of remission during azathioprine therapy in inflammatory bowel disease? Eur J Gastroenterol Hepatol. 2001;13:1073-1076.

11. Persley KM, Present DH. Neutropenia is not required for clinical remission during azathioprine therapy in inflammatory bowel disease. Eur J Gastroenterol Hepatol. 2001;13:1053-1055.

12. Belaiche J, Desager JP, Horsmans Y, Louis E. Therapeutic drug monitoring of azathioprine and 6-mercaptopurine metabolites in Crohn disease. Scand J Gastroenterol. 2001;36:71-76. 